\title{
Editorial
}

\section{Review on epigenetics in cancer gene therapy: series I}

\author{
Cancer Gene Therapy (2005) 12, 663-664. doi:10.1038/sj.cgt.7700884
}

$\mathrm{I}$ n this first series dedicated to Epigenetics, Cancer Gene Therapy has deliberately assembled diverse reviews on topical areas relevant to our understanding of the cancer cell.

In recent years, there has been a surge of interest in the study of cancer and epigenetics, most notably into mechanisms that could be used to modify chromatin architecture in a willing effort to perturb its behavior. Intriguingly, it is technically achievable to modulate the complex architecture and nucleoprotein filament of chromosomes. Studies using histone deacetylase and genomic methylation inhibitors illustrate that chromatin reorganization and decondensation can be achieved over several Mb of DNA. ${ }^{1,2}$ Epigenetic modifiers can rapidly reactivate genes repressed in cancer and are currently being tested in clinical trials. The mechanisms by which these drugs function on the epigenome is not yet clear, although changes in chromatin structure do appear to accompany changing transcriptional activity. ${ }^{3}$ Hyperacetylation of histones and increased chromatin accessibility are just two features associated with gene reactivation. Pharmacological reactivation of silent genes in cancer is an intense area of study and histone deacetylase inhibitors are generally effective against hypomethylated promoter sequences. ${ }^{4}$ The tumor suppressor p16 gene is silenced by DNA methylation and strategies to reactivate include prior cell exposure to trichostatin A and 5-aza-2'deoxycytidine $(5 \mathrm{adC})$ to unlock repressive chromatin structures. ${ }^{5}$ Studies that examine the molecular machineries that localize on hypermethylated sequences have revealed significant changes in methyl-CpG binding domain family members such as MeCP2, MBD1, MBD2 and MBD3 complexes are associated with gene repression in cancer. ${ }^{6-9}$ These and other studies show exquisite changes in DNA methylation, and histone modifications can significantly alter the determinants associated with repression and chromatin conformation.

In a future review article in Cancer Gene Therapy, McNamara and colleagues discuss engineered transcriptional regulators capable of perturbing chromatin to regulate gene expression. The discovery of Cys2His2 motif of zinc-finger proteins (ZFP) and the structural and functional adaptability has made it possible to design modules to dock the major groove of DNA with remarkable specificity. The regulation of gene expression is achieved using engineered ZFP fused to activation or repression domains. Equally impressive is the power of ZFP to modify the chromatin landscape of target genes.
For example, the domains of histone methyltransferases such as Suv39h1 and G9a can be targeted to achieve histone $\mathrm{H} 3 \mathrm{~K} 9$ methylation and associated repression of the VEGF-A promoter. ${ }^{10}$ The authors elegantly examine what is known about targeting ZFP to integrate, assimilate and regulate endogenous chromatin structure and function.

There is now considerable evidence that suggests aberrant $\mathrm{CpG}$ methylation associated with tumor suppressor genes is correlated with carcinogenesis. The protein tyrosine phosphatase (PTP) gene joins the rank of a growing number of identified putative tumor suppressor genes repressed by $\mathrm{CpG}$ methylation. ${ }^{11}$ Repression of genes critical for the control of normal cell growth is a characteristic of neoplasia. The article by Jacob and Motiwala, in pages 665-672 of this issue, examines the role of epigenetic modification of PTP and have put together a comprehensive review of the current state of literature examining silencing. Originally observed in rats fed a folate/methyl-deficient diet, PTP is methylated in preneoplastic nodules and hypothesized to reflect an early tumor marker. ${ }^{12}$ In human lung cancer, PTP transcription is inversely correlated with promoter methylation that is responsive to $5 \mathrm{adC} .{ }^{13}$ The authors also review genome-based therapeutic concepts using epigenetic modifiers to reactivate gene activity.

It is becoming widely appreciated that epigenetic mechanisms crosstalk between DNA methylation, histone modifications and chromatin remodeling machineries and play important roles in regulating nuclear function. RNA interference (RNAi) is an evolutionarily conserved mechanism that involves short double-stranded RNA to target and silence mRNA that share the same sequence identity. Recent developments have revealed that components of the RNAi pathway are also involved in regulating critical aspects of the epigenome. ${ }^{14}$ In support of this view, transcriptional gene silencing can be achieved by the specialized formation of centromeric heterochromatin mediated by RNAi in plants and yeast. ${ }^{15,16}$ In human cells, short interfering RNA have been demonstrated to silence expression by a mechanism mediated by genomic methylation of the target sequence. ${ }^{17,18}$ Although this is the best evidence so far for mammalian RNAi-mediated genomic methylation, the mechanisms by which it occurs are not known. The results to these studies suggest that transcriptional repression can be associated with RNAi and raises important questions about silencing and changes in epigenetic regulation. The article by 
Karagiannis and El-Osta, which will be published in issue 10 , focuses on recent developments in the use of RNAi technologies and potential clinical applications. RNAi has revolutionized our understanding of genome function and has quickly become an essential tool in molecular biology. Already, in the short time since its original discovery, a number of commercially validated siRNAs are now available to translate functional genomics in model biological systems. Furthermore, short interfering RNAs have the potential to treat disease and there is great promise for the gene therapy-based platform as a new therapeutic modality as well as drug target identification and validation.

The purpose of the Epigenetics Series in Cancer Gene Therapy is a response to the extraordinary interest of epigenetics in cancer biology and therapy. The goal is to highlight important developments in the area and it is hoped the review series will continue to develop and mature with emerging research activity.

Assam El-Osta ${ }^{1}$

${ }^{1}$ Epigenetics in Human Health and Disease Laboratory, The Alfred Medical Research and Education Precinct, Baker Medical Research Institute, Victoria, Australia

\section{References}

1. Schmid M, Haaf T, Grunert D. 5-Azacytidine-induced undercondensations in human chromosomes. Hum Genet. 1984;67:257-263.

2. Toth KF, Knoch TA, Wachsmuth M, et al. Trichostatin Ainduced histone acetylation causes decondensation of interphase chromatin. J Cell Sci. 2004;117(Part 18):4277-4287.

3. Gius D, Cui H, Bradbury CM, et al. Distinct effects on gene expression of chemical and genetic manipulation of the cancer epigenome revealed by a multimodality approach. Cancer Cell. 2004;6:361-371.

4. Cameron EE, Bachman KE, Myohanen S, Herman JG, Baylin SB. Synergy of demethylation and histone deacetylase inhibition in the re-expression of genes silenced in cancer. Nat Genet. 1999;21:103-107.

5. Nguyen CT, Weisenberger DJ, Velicescu M, et al. Histone H3-lysine 9 methylation is associated with aberrant gene silencing in cancer cells and is rapidly reversed by 5 -aza- $2^{\prime}-$ deoxycytidine. Cancer Res. 2002;62:6456-6461.

6. El-Osta A, Kantharidis P, Zalcberg JR, Wolffe AP. Precipitous release of methyl-CpG binding protein 2 and histone deacetylase 1 from the methylated human multidrug resistance gene (MDR1) on activation. Mol Cell Biol. 2002;22:1844-1857.

7. Danam RP, Howell SR, Brent TP, Harris LC. Epigenetic regulation of O6-methylguanine-DNA methyltransferase gene expression by histone acetylation and methyl-CpG binding proteins. Mol Cancer Ther. 2005;4:61-69.

8. Magdinier F, Wolffe AP. Selective association of the methyl-CpG binding protein MBD2 with the silent p14/ p16 locus in human neoplasia. Proc Natl Acad Sci USA. 2001;98:4990-4995.

9. Ballestar E, Paz MF, Valle L, et al. Methyl-CpG binding proteins identify novel sites of epigenetic inactivation in human cancer. EMBO J. 2003;22:6335-6345.

10. Snowden AW, Gregory PD, Case CC, Pabo CO. Genespecific targeting of $\mathrm{H} 3 \mathrm{~K} 9$ methylation is sufficient for initiating repression in vivo. Curr Biol. 2002;12:2159-2166.

11. Herman JG, Baylin SB. Gene silencing in cancer in association with promoter hypermethylation. $N$ Engl $J$ Med. 2003;349:2042-2054.

12. Motiwala T, Ghoshal K, Das A, et al. Suppression of the protein tyrosine phosphatase receptor type O gene (PTPRO) by methylation in hepatocellular carcinomas. Oncogene. 2003;22:6319-6331.

13. Motiwala T, Kutay H, Ghoshal K, et al. Protein tyrosine phosphatase receptor-type O (PTPRO) exhibits characteristics of a candidate tumor suppressor in human lung cancer. Proc Natl Acad Sci USA. 2004;101:13844-13849.

14. Lippman Z, Martienssen R. The role of RNA interference in heterochromatic silencing. Nature. 2004;431:364-370.

15. Verdel A, Jia S, Gerber S, et al. RNAi-mediated targeting of heterochromatin by the RITS complex. Science. 2004; 303:672-676.

16. Zilberman D, Cao X, Johansen LK, Xie Z, Carrington JC, Jacobsen SE. Role of Arabidopsis ARGONAUTE4 in RNA-directed DNA methylation triggered by inverted repeats. Curr Biol. 2004;14:1214-1220.

17. Kawasaki H, Taira K. Induction of DNA methylation and gene silencing by short interfering RNAs in human cells. Nature. 2004;431:211-217.

18. Morris KV, Chan SW, Jacobsen SE, Looney DJ. Small interfering RNA-induced transcriptional gene silencing in human cells. Science. 2004;305:1289-1292. 